\title{
On neighbour-distinguishing colourings from lists
}

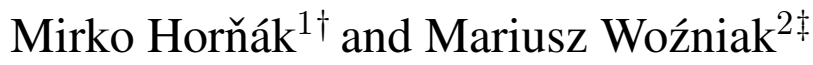 \\ ${ }^{1}$ Institute of Mathematics, Faculty of Science UPJŠ, Košice, Slovakia \\ ${ }^{2}$ Department of Discrete Mathematics, Faculty of Applied Mathematics AGH, Kraków, Poland \\ received $25^{\text {th }}$ May 2009, revised $30^{\text {th }}$ November 2010, $6^{\text {th }}$ February 2012, accepted $10^{\text {th }}$ August 2012.
}

An edge colouring of a graph is said to be neighbour-distinguishing if any two adjacent vertices have distinct sets of colours of their incident edges. In this paper the list version of the problem of determining the minimum number of colours in a neighbour-distinguishing colouring of a given graph is considered.

Keywords: set of colours, list of colours, neighbour-distinguishing edge colouring, list neighbour-dis- tinguishing index, general list neighbour-distinguishing index

\section{Introduction}

Let $G$ be a finite simple graph, let $C$ be a set of colours (in this paper we shall always suppose $C \subseteq \mathbb{N}$ ) and let $f: E(G) \rightarrow C$ be an edge colouring of $G$. The colour set of a vertex $v \in V(G)$ with respect to $f$ is the set $S_{f}(v)$ of colours of edges incident to $v$. The colouring $f$ is neighbour-distinguishing if it distinguishes any two adjacent vertices by their colour sets, i.e., $S_{f}(u) \neq S_{f}(v)$ whenever $u, v \in V(G)$ and $u v \in E(G)$.

As usual, we are interested in the smallest number of colours in a neighbour-distinguishing colouring of $G$. If the optimisation runs over all proper colourings, that number is called the neighbour-distinguishing index of $G$, in symbols ndi $(G)$. In the general case (when colourings are not required to be proper) the corresponding invariant is known as the general neighbour-distinguishing index of $G$ and denoted by $\operatorname{gndi}(G)$.

The main goal of this paper is to analyse the list versions of the above problems. Denote by $\mathcal{P}_{k}(X)$ the set of all $k$-subsets of a set $X$ and by $\mathcal{L}_{k}(G)$ the set of all mappings $L: E(G) \rightarrow \mathcal{P}_{k}(\mathbb{N})$. The set $L(e)$, $L \in \mathcal{L}_{k}(G), e \in E(G)$, is the list of available colours for the edge $e$. Given $L \in \mathcal{L}_{k}(G)$, the graph $G$ is $L$ neighbour-distinguishable provided there is a proper neighbour-distinguishing colouring $f: E(G) \rightarrow \mathbb{N}$ satisfying $f(e) \in L(e)$ for every $e \in E(G)$. The list neighbour-distinguishing index of $G$, in symbols $\operatorname{lndi}(G)$, is the minimum $k$ such that for every $L \in \mathcal{L}_{k}(G)$ the graph $G$ is $L$-neighbour-distinguishable.

\footnotetext{
${ }^{\dagger}$ Email: mirko.hornak@upjs.sk. The work was supported by Science and Technology Assistance Agency under the contract No. APVV-0023-10, by Grant VEGA 1/0652/12 and by the Agency of the Slovak Ministry of Education for the Structural Funds of the EU under the project ITMS:26220120007.

$\ddagger$ Email: mwozniak@uci.agh. edu.pl. The research was partially supported by the Polish Ministry of Science and Higher Education.
}

1365-8050 (c) 2012 Discrete Mathematics and Theoretical Computer Science (DMTCS), Nancy, France 
This invariant is a generalisation of the "classical" edge-choosability $\operatorname{ch}^{\prime}(G)$ of $G$; for the definition of the edge-choosability as well as that of (vertex-) choosability $\operatorname{ch}(G)$ of $G$ see the survey paper [8] by Tuza. Clearly, we have $\operatorname{lndi}(G) \geq \max \left(\operatorname{ndi}(G), \operatorname{ch}^{\prime}(G)\right)$. Recall that according to the List Colouring Conjecture $\operatorname{ch}^{\prime}(G)$ should be equal to $\chi^{\prime}(G)$, the chromatic index of $G$.

Remark. In the study of the neighbour-distinguishing index and the list neighbour-distinguishing index it is useful to realise that adjacent vertices of distinct degrees are trivially distinguished by their colour sets.

A graph $G$ is said to be neighbour-irregular if any two of its vertices of the same degree are nonadjacent. The above remark immediately yields

Proposition 1 If $G$ is a neighbour-irregular graph, then $\operatorname{ndi}(G)=\chi^{\prime}(G)$ and $\operatorname{lndi}(G)=\operatorname{ch}^{\prime}(G)$.

Omitting the requirement that $f$ should be proper we obtain the notions of a generally L-neighbourdistinguishable graph and the general list neighbour-distinguishing index $\operatorname{glndi}(G)$ of the graph $G$.

If $G$ is a disconnected graph with components $G_{1}, \ldots, G_{q}$, then evidently $\operatorname{lndi}(G)=\max \left(\operatorname{lndi}\left(G_{i}\right)\right.$ : $i=1, \ldots, q)$ and $\operatorname{glndi}(G)=\max \left(\operatorname{glndi}\left(G_{i}\right): i=1, \ldots, q\right)$. Therefore, when searching for the neighbour-distinguishing index or general neighbour-distinguishing index, it is sufficient to restrict our attention to connected graphs.

Observe that $\operatorname{lndi}\left(K_{1}\right)=\operatorname{glndi}\left(K_{1}\right)=0$ and that the graph $K_{2}$ does not have any neighbourdistinguishing colouring (either proper or not). So, we shall consider only connected graphs with at least three vertices.

In Section 2 we show that the list neighbour-distinguishing index is equal to the neighbour-distinguishing index for graphs in several classes, namely cycles, trees and unbalanced complete bipartite graphs. This leads us to

Conjecture 2 If $G$ is a connected graph with $|V(G)| \geq 3$, then $\operatorname{lndi}(G)=\operatorname{ndi}(G)$.

Remark. From Proposition 1 it follows that for neighbour-irregular graphs Conjecture 2 and the List Colouring Conjecture are equivalent.

In Section 3 we prove that the general list neighbour-distinguishing index of a tree is at most 3 and we exhibit a tree $T$ with $\operatorname{glndi}(G)>\operatorname{gndi}(G)$.

Given $p, q \in \mathbb{Z}$, let $[p, q]:=\bigcup_{i=p}^{q}\{i\}$ be the integer interval bounded by $p$ and $q$. The concatenation of finite sequences $A=\left(a_{1}, \ldots, a_{m}\right)$ and $B=\left(b_{1}, \ldots, b_{n}\right)$ is the sequence $A B:=\left(a_{1}, \ldots, a_{m}, b_{1}, \ldots, b_{n}\right)$. Concatenating finite sequences is clearly associative and we shall use $\prod_{i=1}^{k} A^{(i)}$ for the result of (successive) concatenation of finite sequences $A^{(1)}, \ldots, A^{(k)}$. So, if $A^{(i)}=\left(a_{1}^{(i)}, \ldots, a_{m^{(i)}}^{(i)}\right)$, then $\prod_{i=1}^{k} A^{(i)}=$ $\left(a_{1}^{(1)}, \ldots, a_{m^{(1)}}^{(1)}, \ldots, a_{1}^{(k)}, \ldots, a_{m^{(k)}}^{(k)}\right)$. For $k \in \mathbb{Z}$ we denote by $(k)_{2}$ the (unique) $l \in[2,3]$ satisfying $k \equiv l(\bmod 2)$.

Recall that a cycle $C$ of length $l \geq 3$ in a graph $G$ can be coded using a sequence $\prod_{i=1}^{l+1}\left(v_{i}\right)$ of its consecutive vertices so that $V(C)=\left\{v_{i}: i \in[1, l]\right\}$ and $E(C)=\left\{v_{i} v_{i+1}: i \in[1, l]\right\}$ (with $v_{l+1}=v_{1}$ ).

\section{Proper colourings}

The neighbour-distinguishing index of a graph was introduced by Zhang et al. in [9], where the authors found (among other things) the value of this invariant for cycles $\left(C_{n}\right.$ denotes an $n$-vertex cycle) and trees:

Proposition 3 Let $n$ be an integer, $n \geq 3$. 
(1) If $n=5$, then $\operatorname{ndi}\left(C_{n}\right)=5$.

(2) If $n \not \equiv 0(\bmod 3), n \neq 5$, then $\operatorname{ndi}\left(C_{n}\right)=4$.

(3) If $n \equiv 0(\bmod 3)$, then $\operatorname{ndi}\left(C_{n}\right)=3$.

Proposition 4 If $T$ is a tree with $|V(T)| \geq 3$, then $\Delta(T) \leq \operatorname{ndi}(T) \leq \Delta(T)+1$. Moreover, $\operatorname{ndi}(T)=$ $\Delta(T)+1$ if and only if $T$ has a pair of adjacent vertices of degree $\Delta(T)$.

In the above introductory paper it is conjectured that $\operatorname{ndi}(G) \leq \Delta(G)+2$ for any connected graph $G \notin\left\{K_{2}, C_{5}\right\}$. The conjecture was confirmed by Balister et al. in [1] for bipartite graphs and for graphs $G$ with $\Delta(G)=3$. Edwards et al. in [2] showed even that $\operatorname{ndi}(G) \leq \Delta(G)+1$ if $G$ is bipartite, planar, and of maximum degree $\Delta(G) \geq 12$.

In this section we determine the list neighbour-distinguishing index for graphs in some classes with a simple structure.

\subsection{Cycles}

We suppose that $E\left(C_{n}\right)=\left\{e_{i}: i \in[1, n]\right\}$ and that $e_{i}$ is adjacent to $e_{i+1}$ for every $i \in[1, n]$ with indices taken modulo $n$ (this usual convention will be used throughout the whole paper without explicitly mentioning it in what follows).

Proposition 5 Let $n$ be an integer, $n \geq 3$.

(1) If $n=5$, then $\operatorname{lndi}\left(C_{n}\right)=\operatorname{ndi}\left(C_{n}\right)=5$.

(2) If $n \not \equiv 0(\bmod 3), n \neq 5$, then $\operatorname{lndi}\left(C_{n}\right)=\operatorname{ndi}\left(C_{n}\right)=4$.

(3) If $n \equiv 0(\bmod 3)$, then $\operatorname{lndi}\left(C_{n}\right)=\operatorname{ndi}\left(C_{n}\right)=3$.

Proof: Because of Proposition 3 we shall be done by proving the inequality $\operatorname{lndi}\left(C_{n}\right) \leq \operatorname{ndi}\left(C_{n}\right)$ (the reverse inequality being obvious).

(1) The statement is trivial.

(2) It suffices to show that for any $L \in \mathcal{L}_{4}\left(C_{n}\right)$ there is a proper neighbour-distinguishing colouring $f: E\left(C_{n}\right) \rightarrow \mathbb{N}$ satisfying $f\left(e_{i}\right) \in L\left(e_{i}\right)$ for every $i \in[1, n]$.

If all lists are the same, the existence of $f$ follows from the fact that $\operatorname{ndi}\left(C_{n}\right)=4$. Otherwise there are two adjacent edges of $C_{n}$ with distinct lists. Without loss of generality we may suppose that $L\left(e_{1}\right) \neq L\left(e_{n}\right)$, which implies $L\left(e_{1}\right) \backslash L\left(e_{n}\right) \neq \emptyset$. Now determine $f\left(e_{i}\right)$ in the following way: Choose $f\left(e_{1}\right) \in L\left(e_{1}\right) \backslash L\left(e_{n}\right)$ and $f\left(e_{2}\right) \in L\left(e_{2}\right) \backslash\left\{f\left(e_{1}\right)\right\}$. If $j \in[3, n-2]$ and $f\left(e_{i}\right)$ is known for all $i \in[1, j-1]$, take $f\left(e_{j}\right) \in L\left(e_{j}\right) \backslash\left\{f\left(e_{j-2}\right), f\left(e_{j-1}\right)\right\}$. To finish, let $f\left(e_{n-1}\right) \in$ $L\left(e_{n-1}\right) \backslash\left\{f\left(e_{n-3}\right), f\left(e_{n-2}\right), f\left(e_{1}\right)\right\}$ and $f\left(e_{n}\right) \in L\left(e_{n}\right) \backslash\left\{f\left(e_{n-2}\right), f\left(e_{n-1}\right), f\left(e_{1}\right), f\left(e_{2}\right)\right\}$ (which is possible because $\left.f\left(e_{1}\right) \notin L\left(e_{n}\right)\right)$.

(3) An edge colouring $f: E\left(C_{n}\right) \rightarrow \mathbb{N}$ is proper and neighbour-distinguishing if and only if $f\left(e_{i+1}\right) \neq$ $f\left(e_{i}\right) \neq f\left(e_{i+2}\right)$ for every $i \in[1, n]$. Let $F_{n}$ be the graph with $V\left(F_{n}\right)=E\left(C_{n}\right)$ and $E\left(F_{n}\right)=$ $\left\{e_{i} e_{i+1}: i \in[1, n]\right\} \cup\left\{e_{i} e_{i+2}: i \in[1, n]\right\}$. Thus $F_{n}$ is the square of the line graph of $C_{n}$ and, as the 
line graph of $C_{n}$ is $C_{n}$ itself, $F_{n} \cong C_{n}^{2}\left(F_{n}\right.$ is isomorphic to $\left.C_{n}^{2}\right)$. Therefore, $\operatorname{lndi}\left(C_{n}\right)=\operatorname{ch}\left(F_{n}\right)=$ $\operatorname{ch}\left(C_{n}^{2}\right)$.

Since $n \equiv 0(\bmod 3)$, say $n=3 k$, it is clear that the graph $F_{n}$ is an edge-disjoint union of the cycle $\left(e_{2}\right) \prod_{j=1}^{k}\left(e_{3 j-2}, e_{3 j}, e_{3 j+2}\right)$ of length $3 k$ and $k$ vertex-disjoint cycles $\left(e_{3 j-1}, e_{3 j}, e_{3 j+1}, e_{3 j-1}\right)$ of length 3 with $j \in[1, k]$. Graphs with such a structure were studied by Fleischner and Stiebitz in [3], where it is proved that $\operatorname{ch}\left(F_{n}\right)=3$. So, by Proposition 3. (3), we have $\operatorname{lndi}\left(C_{n}\right)=\operatorname{ch}\left(F_{n}\right)=$ $\operatorname{ndi}\left(C_{n}\right)=3$.

\subsection{Trees}

By Proposition 4, there are two categories of trees: Category $k$ consists of trees $T$ with ndi $(T)=\Delta(T)-$ $1+k, k=1,2$. Thus, a tree $T$ is of Category 2 if and only if it has a pair of adjacent vertices of degree $\Delta(T)$.

Theorem 6 If $T$ is a tree with $|V(T)| \geq 3$, then $\operatorname{lndi}(T)=\operatorname{ndi}(T)$.

Proof: Consider the following algorithm that enables us to find a neighbour-distinguishing colouring of a given tree $T$ of a given category with colours belonging to lists of an appropriate cardinality.

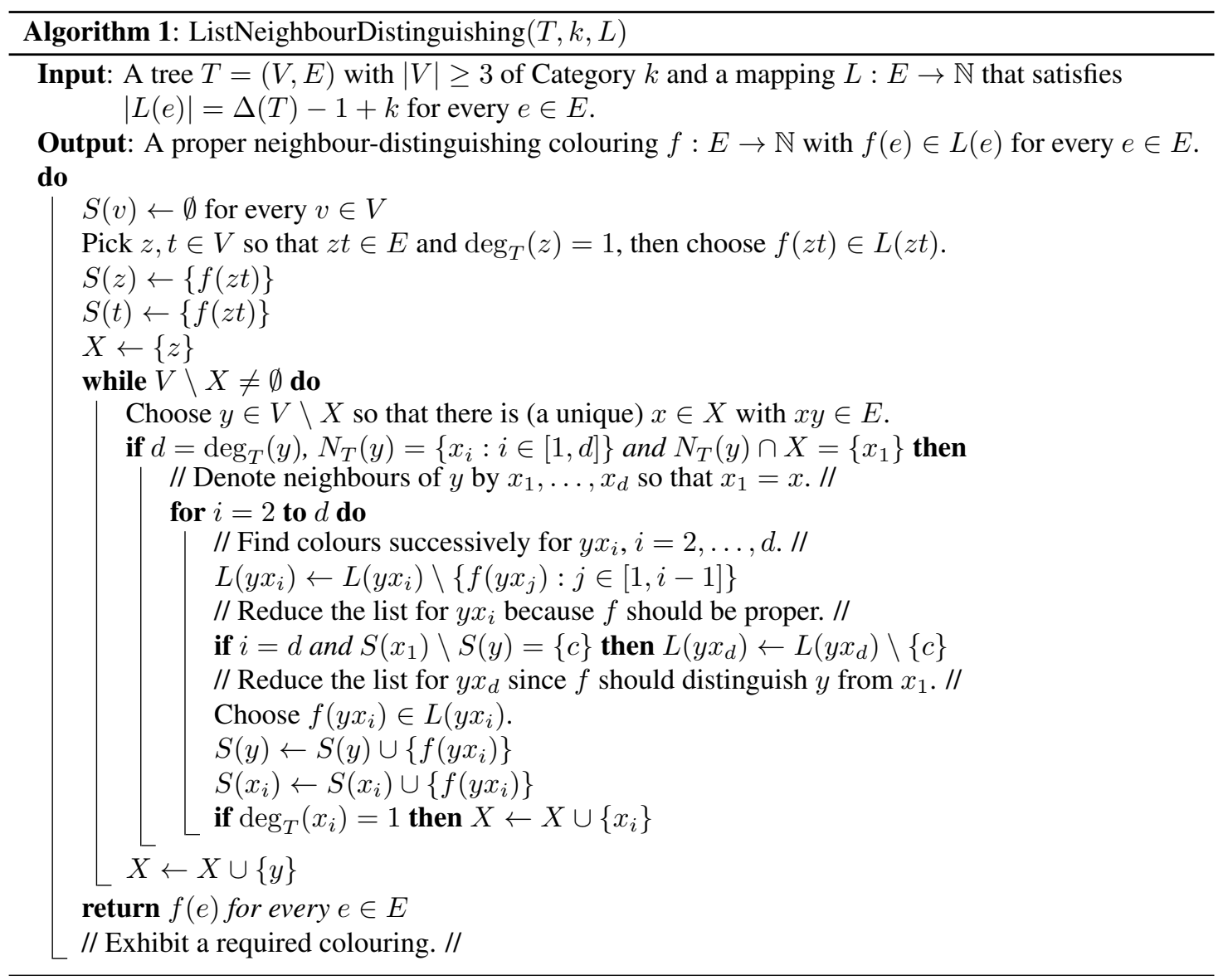


The algorithm builds step by step colour sets $S(v)$ of vertices $v \in V$ by assigning colours to all uncoloured edges incident to $v$. It starts with colouring an arbitrary pendant edge of $T$ so that the (unique) pendant vertex of this edge is the first vertex of the set $X$ of all vertices of $T$ with currently finished colour sets. If $X \neq V$, the subgraph of $T$ induced by all currently coloured edges has a leaf out of $X$ (an extension vertex). A successive colouring of all edges (not coloured yet) incident to the extension vertex extends $X$ by all pendant neighbours (out of $X$ ) of the extension vertex and, finally, by the extension vertex itself.

To see that the algorithm is correct it is sufficient to use the following two facts:

a) No list is reduced to the empty set. With $k=1$ the worst case (the reduction to a singleton) can apply if either $\operatorname{deg}_{T}(y)=\Delta(T)$ or $\operatorname{deg}_{T}(x)=\Delta(T)-1=\operatorname{deg}_{T}(y)$. If $k=2$, such a situation can appear provided that $\operatorname{deg}_{T}(x)=\Delta(T)=\operatorname{deg}_{T}(y)$.

b) If a vertex $y$ is added to $X$ and $X \cup\{y\} \neq V$, then the subgraph of $T$ induced by all currently coloured edges is a subtree of $T$, all its non-leaves are in $X$ and at least one of its leaves is not a leaf of the whole $T$ (so that it can be chosen as a new $y$ and guarantees that $d \geq 2$ in the corresponding for loop).

\subsection{Unbalanced complete bipartite graphs}

Unbalanced complete bipartite graphs also are in favour of Conjecture 2.

Theorem 7 If $m, n$ are positive integers with $m<n$, then $\operatorname{lndi}\left(K_{m, n}\right)=\operatorname{ndi}\left(K_{m, n}\right)=n$.

Proof: It is well known that $\chi^{\prime}\left(K_{m, n}\right)=n$. Since $K_{m, n}$ is neighbour-irregular, by Proposition 1 we have $\operatorname{ndi}\left(K_{m, n}\right)=\chi^{\prime}\left(K_{m, n}\right)=n$.

On the other hand, Janssen in [7] proved that $\operatorname{ch}^{\prime}\left(K_{m, n}\right)=n$; so, again by Proposition $1, \operatorname{lndi}\left(K_{m, n}\right)=$ $\operatorname{ch}^{\prime}\left(K_{m, n}\right)=n$ and we are done.

\section{General colourings}

The general neighbour-distinguishing index was introduced by Győri et al. in [4], where the authors proved that $\operatorname{gndi}(G) \leq 2\left\lceil\log _{2} \chi(G)\right\rceil+1$ for any graph $G$ having no isolated edges. The result was improved by Györi and Palmer who proved in [5] that $\chi(G) \geq 3$ implies gndi $(G)=\left\lceil\log _{2} \chi(G)\right\rceil+1$ (in the case $\log _{2} \chi(G) \notin \mathbb{Z}$ the same expression was obtained independently by Horňák and Soták in [6]).

The analysis of the general list neighbour-distinguishing index seems to be a bit more complicated than that of the list neighbour-distinguishing index. Indeed, we know the exact values just for paths and cycles, for trees we only have an upper bound equal to 3. Also, an analogue of Conjecture 2 fails.

\subsection{Paths and cycles}

We shall see that, as in the case of proper colourings, when passing to the list version the value of the parameter does not change in the classes of paths and cycles. 
Proposition 8 Let $n$ be an integer, $n \geq 3$.

(1) If $n \equiv 1(\bmod 2)$, then $\operatorname{glndi}\left(P_{n}\right)=\operatorname{gndi}\left(P_{n}\right)=2$.

(2) If $n \equiv 0(\bmod 2)$, then $\operatorname{glndi}\left(P_{n}\right)=\operatorname{gndi}\left(P_{n}\right)=3$.

Proof: The values of $\operatorname{gndi}\left(P_{n}\right)$ come from [4] so that we only need to show that $\operatorname{glndi}\left(P_{n}\right)=\operatorname{gndi}\left(P_{n}\right)$.

An edge colouring $f: E\left(P_{n}\right) \rightarrow \mathbb{N}$ is neighbour-distinguishing if and only if $f\left(e_{1}\right) \neq f\left(e_{2}\right), f\left(e_{i}\right) \neq$ $f\left(e_{i+2}\right)$ for every $i \in[1, n-3]$ and $f\left(e_{n-2}\right) \neq f\left(e_{n-1}\right)$. Let $G_{n}$ be the graph with $V\left(G_{n}\right)=E\left(P_{n}\right)$ and $E\left(G_{n}\right)=\left\{e_{1} e_{2}\right\} \cup\left\{e_{i} e_{i+2}: i \in[1, n-3]\right\} \cup\left\{e_{n-2} e_{n-1}\right\}$. If $n \geq 4(n=2 k$ or $n=2 k+1$ with $k \in \mathbb{Z}$, $k \geq 2$ ), then $G_{n} \cong C_{n-1}$. Indeed, the following are cycles in $G_{n}:\left(e_{1}\right) \prod_{i=1}^{k-1}\left(e_{2 i}\right)\left[\prod_{i=1}^{k}\left(e_{2 k+1-2 i}\right)\right]$ (if $n=2 k$ ) and $\left(e_{1}\right) \prod_{i=1}^{k}\left(e_{2 i}\right)\left[\prod_{i=1}^{k}\left(e_{2 k+1-2 i}\right)\right]$ (if $\left.n=2 k+1\right)$. Therefore, glndi $\left(P_{n}\right)=\operatorname{ch}\left(C_{n-1}\right)$ and we are done by using the well-known fact that $\operatorname{ch}\left(C_{l}\right)=(l)_{2}$. Finally, $G_{3} \cong P_{2}$, hence $\operatorname{glndi}\left(P_{3}\right)=$ $\operatorname{ch}\left(P_{2}\right)=2=\operatorname{gndi}\left(P_{3}\right)$.

Proposition 9 Let $n$ be an integer, $n \geq 3$.

(1) If $n \equiv 0(\bmod 4)$, then $\operatorname{glndi}\left(C_{n}\right)=\operatorname{gndi}\left(C_{n}\right)=2$.

(2) If $n \neq 0(\bmod 4)$, then $\operatorname{glndi}\left(C_{n}\right)=\operatorname{gndi}\left(C_{n}\right)=3$.

Proof: Similarly as in the preceding proposition, we only have to prove that $\operatorname{glndi}\left(C_{n}\right)=\operatorname{gndi}\left(C_{n}\right)$ (since gndi $\left(C_{n}\right)$ is known from [4]).

An edge colouring $f: E\left(C_{n}\right) \rightarrow \mathbb{N}$ is neighbour-distinguishing if and only if $f\left(e_{i}\right) \neq f\left(e_{i+2}\right)$ for every $i \in[1, n]$. Let $H_{n}$ be the graph with $V\left(H_{n}\right)=E\left(C_{n}\right)$ and $E\left(H_{n}\right)=\left\{e_{i} e_{i+2}: i \in[1, n]\right\}$; then $\operatorname{glndi}\left(C_{n}\right)=\operatorname{ch}\left(H_{n}\right)$.

If $n$ is odd, $n=2 k+1$, then $\left(e_{2 k}\right) \prod_{i=0}^{k}\left(e_{2 i+1}\right)\left[\prod_{i=1}^{k}\left(e_{2 i}\right)\right]$ is a cycle in $H_{n}, H_{n} \cong C_{n}$ and $\operatorname{glndi}\left(C_{n}\right)=\operatorname{ch}\left(C_{n}\right)=3$. If $n$ is even, $n=2 k, k \geq 3$, then $\left(e_{2 k-2+j}\right) \prod_{i=0}^{k-1}\left(e_{j+2 i}\right), j=1,2$, is a cycle in $H_{n}$, hence $H_{n} \cong 2 C_{k}$ and $\operatorname{glndi}\left(C_{n}\right)=\operatorname{ch}\left(2 C_{k}\right)=\operatorname{ch}\left(C_{k}\right)=(k)_{2}$. Finally, $H_{4} \cong 2 P_{2}$, and $\operatorname{glndi}\left(H_{4}\right)=\operatorname{ch}\left(2 P_{2}\right)=\operatorname{ch}\left(P_{2}\right)=2$.

\subsection{Trees}

The following two results are taken from [4].

Theorem 10 If $G$ is a connected bipartite graph with $|V(G)| \geq 3$, then $2 \leq \operatorname{gndi}(G) \leq 3$.

Proposition 11 For any graph $G$ the following statements are equivalent:

(1) $\operatorname{gndi}(G)=2$.

(2) $G$ is bipartite and there is a bipartition $\left\{X_{1} \cup X_{2}, Y\right\}$ of $V(G)$ such that $X_{1} \cap X_{2}=\emptyset$ and any vertex of $Y$ has at least one neighbour in both $X_{1}$ and $X_{2}$.

Proposition 12 If $T$ is a tree with $|V(T)| \geq 3$, then $\operatorname{gndi}(T)=2$ if and only if the distance between any two leaves of $T$ is even. 
Proof: If $\operatorname{gndi}(T)=2$, by Proposition 11 we know that there is a bipartition $\left\{X_{1} \cup X_{2}, Y\right\}$ of $V(T)$ such that $X_{1} \cap X_{2}=\emptyset$ and any vertex of $Y$ has at least one neighbour in both $X_{1}$ and $X_{2}$. Thus $\operatorname{deg}_{T}(y) \geq 2$ for every $y \in Y$, all leaves of $T$ are in $X_{1} \cup X_{2}$ and the distance between any two of them is even.

Now suppose that the distance between any two leaves of $T$ is even. Let $\{X, Y\}$ be the bipartition $\{X, Y\}$ of $V(T)$ with $X$ containing all leaves of $T$. Pick $x_{0} \in X$ and let $\left\{X_{1}, X_{2}\right\}$ be the decomposition of $X$ determined as follows: $x \in X_{i} \stackrel{\text { df. }}{\Leftrightarrow} d_{T}\left(x_{0}, x\right) \equiv 2 i(\bmod 4), i=1,2$. Consider a vertex $y \in Y$ and its neighbour $x^{\prime}$ lying on the (unique) path joining $y$ to $x_{0}$. Since $y$ is not a leaf, it has a neighbour $x^{\prime \prime} \neq x^{\prime}$. Clearly, $d_{T}\left(x_{0}, x^{\prime \prime}\right)=d_{T}\left(x_{0}, x^{\prime}\right)+2$, which means that one of $x^{\prime}, x^{\prime \prime}$ is in $X_{1}$ and the other in $X_{2}$. By Proposition 11 then $\operatorname{gndi}(T)=2$.

Theorem 13 If $T$ is a tree with $|V(T)| \geq 3$, then $\operatorname{glndi}(T) \leq 3$.

Proof: We proceed by induction on the lexicographical ordering of pairs $(\operatorname{diam}(T),|V(T)|)$, where $\operatorname{diam}(T)$ is the diameter of $T$. From $|V(T)| \geq 3$ we know that $\operatorname{diam}(T) \geq 2$. If $\operatorname{diam}(T)=2$, then $T$ is a star and glndi $(T)=2$ (it is sufficient to colour the second coloured edge with a colour that is different from the first used colour).

So, suppose that $\operatorname{diam}(T) \geq 3$ and $\operatorname{glndi}\left(T^{\prime}\right) \leq 3$ for every tree $T^{\prime}$ with the pair $\left(\operatorname{diam}\left(T^{\prime}\right),\left|V\left(T^{\prime}\right)\right|\right)$ lexicographically smaller than the pair $(\operatorname{diam}(T),|V(T)|)$. Let $x$ be an endvertex (a leaf) of a longest path in $T$ and let $y$ be the unique neighbour of $x$. Further, let $z$ be the unique non-leaf neighbour of $y$.

Consider a mapping $L \in \mathcal{L}_{3}(T)$, the tree $T^{\prime}:=T-x$ and the mapping $L^{\prime}:=L \backslash\{(x y, L(x y))\} \in$ $\mathcal{L}_{3}\left(T^{\prime}\right)$. By the induction hypothesis there is a neighbour-distinguishing colouring $f^{\prime}: E\left(T^{\prime}\right) \rightarrow \mathbb{N}$ with $f^{\prime}(e) \in L^{\prime}(e)=L(e)$ for every $e \in E\left(T^{\prime}\right)$. Let $f: E(T) \rightarrow \mathbb{N}$ be an extension of $f^{\prime}$ with $f(x y) \in L(x y)$ that has the following two properties: if $S_{f^{\prime}}(z) \backslash S_{f^{\prime}}(y)=\left\{c_{1}\right\}$, then $f(x y) \neq c_{1}$; if $S_{f^{\prime}}(y)=\left\{c_{2}\right\}$ (which means that $\operatorname{deg}_{T^{\prime}}(y)=1$ ), then $f(x y) \neq c_{2}$. Since $|L(x y)|=3, f$ does exist. Clearly, $f$ is neighbour-distinguishing, the tree $T$ is $L$-neighbour-distinguishable and $\operatorname{gln} \operatorname{di}(T) \leq 3$.

The rest of this subsection is devoted to showing that an analogue of Conjecture 2 , in which the list neighbour-distinguishing index is replaced by the general list neighbour-distinguishing index, fails.

Let $T$ and $L$ be the tree and the mapping from $\mathcal{L}_{2}(T)$, respectively, that are presented in Figure 1 . The distance between any two leaves in $T$ is even, hence, by Proposition 12 , gndi $(T)=2$. Let us prove that $T$ is not $L$-neighbour-distinguishable (so that, by Theorem 13 , glndi $(T)=3$ ). Suppose, on the contrary, that $f$ is a neighbour-distinguishing colouring of $T$ with $f(e) \in L(e)$ for every $e \in E(T)$.

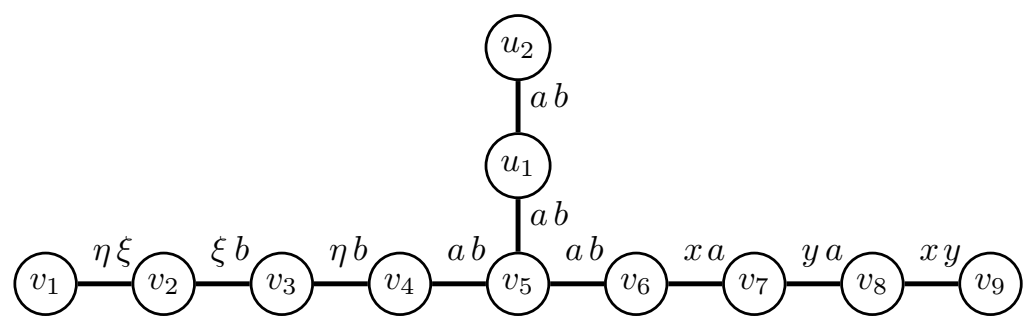

Fig. 1: A tree $T$ with $2=\operatorname{gndi}(T)<\operatorname{glndi}(T)=3$. 
Case 1: $\left|S_{f}\left(v_{5}\right)\right|=1$

Suppose first that $S_{f}\left(v_{5}\right)=\{a\}$. Then $S_{f}\left(v_{6}\right) \neq S_{f}\left(v_{7}\right)$ implies $f\left(v_{7} v_{8}\right)=y$ and, consequently, $f\left(v_{8} v_{9}\right)=x$. Now, however, with $f\left(v_{6} v_{7}\right)=x$ we obtain $S_{f}\left(v_{7}\right)=\{x, y\}=S_{f}\left(v_{8}\right)$, while $f\left(v_{6} v_{7}\right)=a$ yields $S_{f}\left(v_{5}\right)=\{a\}=S_{f}\left(v_{6}\right)$, a contradiction in both cases. If $S_{f}\left(v_{5}\right)=\{b\}$, we get a contradiction by a "symmetrical" reasoning on the "left-hand side" of $T$.

Case 2: $S_{f}\left(v_{5}\right)=\{a, b\}$

Now it follows from $S_{f}\left(v_{5}\right) \neq S_{f}\left(u_{1}\right)$ and $L\left(v_{5} u_{1}\right)=L\left(u_{1} u_{2}\right)=\{a, b\}=S_{f}\left(v_{5}\right)$ that $\left|S_{f}\left(u_{1}\right)\right|=1$. As a consequence of $d_{T}\left(u_{2}\right)=1$ we have also $\left|S_{f}\left(u_{2}\right)\right|=1$. Then, however, from $f\left(u_{1} u_{2}\right) \in S_{f}\left(u_{1}\right) \cap S_{f}\left(u_{2}\right)$ we obtain $S_{f}\left(u_{1}\right)=S_{f}\left(u_{2}\right)$, a contradiction.

\section{Acknowledgements}

The authors are indebted to an anonymous referee whose well-founded comments helped to improve the presentation of obtained results. They are thankful to Zsolt Tuza for pointing out that Janssen's theorem also yields a support for Conjecture 2

\section{References}

[1] P.N. Balister, E. Győri, J. Lehel, R.H. Schelp, Adjacent vertex distinguishing edge-colorings, SIAM J. Discrete Math. 21 (2007) 237-250.

[2] K. Edwards, M. Horňák, M. Woźniak, On the neighbour-distinguishing index of a graph, Graphs Combin. 22 (2006) 341-350.

[3] H. Fleischner, M. Stiebitz, A solution to a colouring problem of P. Erdôs, Discrete Math. 101 (1992) 39-48.

[4] E. Győri, M. Horňák, C. Palmer, M. Woźniak, General neighbour-distinguishing index of a graph, Discrete Math. 308 (2008) 827-831.

[5] E. Győri, C. Palmer, A new type of edge-derived vertex colorings, Discrete Math. 309 (2009), $6344-$ 6352.

[6] M. Horňák, R. Soták, General neighbour-distinguishing index via chromatic number, Discrete Math. 310 (2010), 1733-1736.

[7] J.C.M. Janssen, The Dinitz problem solved for rectangles, Bull. Amer. Math. Soc. 29 (1993) $243-$ 249.

[8] Zs. Tuza, Graph colorings with local constraints—a survey, Discuss. Math. Graph Theory 17 (1997) 161-228.

[9] Z. Zhang, L. Liu, J. Wang, Adjacent strong edge coloring of graphs, Appl. Math. Lett. 15 (2002) 623-626. 